Smith School of Enterprise and the Environment, School of Geography and the Environment, University of Oxford, Oxford, UK

2 Future of Cooling Programme, Oxford Martin School, University of Oxford, Oxford, UK

3 Nuffield Department of Primary Care Health Sciences, University of Oxford, Oxford, UK

Correspondence to: R Perera rafael.perera@phc.ox.ac.uk

Cite this as: BMJ 2021;375:n2438 http://dx.doi.org/10.1136/bmj.n2438

Published: 07 October 2021

\section{Health risks of extreme heat}

\author{
Risks are high, and rising in line with global temperatures \\ Radhika Khosla, ${ }^{1,2}$ Anant Jani, ${ }^{2}$ Rafael Perera ${ }^{2,3}$
}

Record breaking heat events, once rare, are now common. ${ }^{1}$ An extra 475 million exposures to heatwaves (that is, one person experiencing three days or more of extreme heat) were observed globally in 2019 compared with 1986-2005. ${ }^{2}$ Clear evidence links the intensity and frequency of heat extremes to human induced global warming, which also increases the probability of compound events such as concurrent heatwaves and droughts. ${ }^{3}$ The implications are serious: extreme heat has a substantial impact on all 17 UN sustainable development goals-including zero hunger, good health and wellbeing for all, climate action, and reduced inequalities ${ }^{4}$-directly affecting both people and the planet. ${ }^{5}$

Important physiological changes occur in response to high temperatures, and these are amplified by humidity. For example, blood flow is redistributed to the skin, sweating increases, ${ }^{6}$ and adjustments occur to the circulatory (such as vasodilation), nervous (autonomic activation), and respiratory systems (in response to increased oxygen demand) to maintain optimal body temperature; changes also occur in the renal system to maintain fluid and electrolyte balance and organ perfusion. ${ }^{7}$

Exceeding thermoregulatory adaptations leads to overheating with various adverse health consequences, including cardiopulmonary problems in high risk groups (older adults, young children, people with chronic conditions, athletes, and outdoor workers ${ }^{8}$ ) and increased cardiovascular mortality. ${ }^{9}$ Severe heat disrupts sleep, impairs cognitive performance, and is associated with increased risk of suicide or hospital admission for mental illness. ${ }^{10}$

The Global Burden of Disease study estimated that about $20 \%$ of all deaths attributable to extreme heat and cold in 2019 (356 00o/1.7 million) were caused by high temperatures. ${ }^{11}$ This does not account for indirect effects such as crop failure leading to hunger or reduction in national gross domestic product (GDP) because of potential lost labour capacity-India and Indonesia are estimated to have lost $4-6 \%$ of GDP in 2019. ${ }^{2}$ Critically, although direct causes of death such as stroke or cardiomyopathy had a J-shaped relation with temperature in this study (increased risk at both extremes), the risk of external causes of death such as homicide or suicide rose monotonically as temperatures increased. ${ }^{11}$ If current trends continue, heat thresholds for maintaining health are likely to be exceeded far more often, ${ }^{3}$ resulting in further heat related morbidity and mortality. ${ }^{7}$

Healthcare systems globally must adapt to increased healthcare demand in a warming world. Those already contending with seasonal fluctuations-such as "winter pressures" in colder western countries-are now faced with severe heat emerging as new "summer pressures." 12 Healthcare systems in hotter low and middle income countries will fare even worse as already overstretched services fail to cope with increased demand. ${ }^{13}$

\section{Prevention}

Heat related vulnerabilities are exacerbated by chronic conditions such as cardiovascular disease, respiratory diseases, diabetes, and hypertension and by behaviours such as alcohol consumption, misuse of illegal substances, and inadequate fluid intake. ${ }^{7}$ All are exacerbated by social and economic inequality. Long term population-based interventions targeting the modifiable risk factors underlying these conditions and behaviours could therefore reduce the negative health effects of extreme heat and should be prioritised. ${ }^{14}$ Implementing prevention strategies linked to lifestyle changes (in diet or physical activity, for example) is likely to be more cost effective than managing acute healthcare needs linked to extreme heat. A policy focus on prevention would increase health system resilience by improving population health and reducing demand for healthcare. ${ }^{14}$

In addition, broader and more holistic adaptation is required. Examples include heat-health action plans coordinated across government departments and implemented with clear communication strategies, real time surveillance, and local and national evaluation ${ }^{15}$; introducing natural cooling solutions to urban built environments such as increasing use of tree cover, water bodies, and reflective surfaces that mitigate heat exposure in cities $^{3}$; and promoting cooling behavioural strategies such as changing dress codes and times of work. ${ }^{16}$

Importantly, these low energy solutions help reduce health risks without worsening climate change. In contrast, energy intensive air conditioning, likely to be the world's primary heat reduction strategy, exacerbates global warming and drives further extreme heat events, ${ }^{5}$ while remaining unaffordable for the estimated two to five billion people who will be exposed to heat stress by 2050. ${ }^{17}$

Ensuring equitable solutions to the health harms of extreme heat is crucial, particularly in low income countries in southern Asia and Africa, where climate effects are likely to be most devastating and where mitigation options are limited and often unsustainable. ${ }^{18}$ Even high income countries can expect an unequal distribution of heat related harm, driven by existing socioeconomic inequality. ${ }^{19}$ Equitable responses to severe heat include measures such as prioritising populations at greatest risk for early intervention, including by appointing local "heat officers" in urban centres. ${ }^{20}$ 
Professionals in healthcare, public health, and social care must come together to ensure that we place vulnerable populations, communities, and individuals at the heart of our collective efforts in planetary stewardship.

Competing interests: We have read and understood the BMJ policy on declaration of interests and have no interests to declare.

Provenance and peer review: Not commissioned; externally peer reviewed.

1 Sherwood SC. Adapting to the challenges of warming. Science 2020;370:782-3.

https://www. sciencemag.org/lookup/doi/10.1126/science.abe4479.

doi: 10.1126/science.abe4479 pmid: 33184202

2 Lancet Countdown on Health and Climate Change. Explore our data. 2020.

https://www.lancetcountdown.org/data-platform/climate-change-impacts-exposures-and-vulnerability/1-1-health-and-heat/1-1-2-health-and-exposure-to-warming

3 Nakic'enovic ${ }^{\prime} \mathrm{N}^{`}$, Davidson O, Davis G, et al. Summary for policymakers. In: International Panel on Climate Change. Climate change 2014: mitigation of climate change. Cambridge University Press, 2014:1-161. https://ebooks.cambridge.org/ref/id/CB09781107415416A011

4 United Nations. The 17 goals. https://sdgs.un.org/goals

5 Khosla R, Miranda ND, Trotter PA, etal. Cooling for sustainable development. Nat Sustain 2021:4:201-8doi: 10.1038/s41893-020-00627-w

6 Kenny GP, Jay 0 . Thermometry, calorimetry, and mean body temperature during heat stress. In: Comprehensive physiology. Wiley, 2013: 1689-719. https://onlinelibrary.wi-

ley.com/doi/10.1002/cphy.c130011doi: 10.1002/cphy.c130011

7 Ebi KL, Capon A, Berry P, etal. Hot weather and heat extremes: health risks. Lancet 2021;398:698-708. doi: 10.1016/S0140-6736(21)01208-3 pmid: 34419205

8 Centers for Disease Control and Prevention. Protecting vulnerable groups from extreme heat. 2017. https://www.cdc.gov/disasters/extremeheat/specificgroups.html

9 Cheng J, Xu Z, Bambrick H, etal. Cardiorespiratory effects of heatwaves: A systematic review and meta-analysis of global epidemiological evidence. Environ Res 2019;177:108610. doi: 10.1016/j.envres.2019.108610 pmid: 31376629

10 Thompson R, Hornigold R, Page L, Waite T. Associations between high ambient temperatures and heat waves with mental health outcomes: a systematic review. Public Health2018;161:171-91. doi: 10.1016/j.puhe.2018.06.008 pmid: 30007545

11 Burkart KG, Brauer M, Aravkin AY, etal. Estimating the cause-specific relative risks of non-optimal temperature on daily mortality: a two-part modelling approach applied to the Global Burden of Disease Study. Lancet2021;398:685-97. doi: 10.1016/S0140-6736(21)01700-1 pmid: 34419204

12 lacobucci G. "No respite" for NHS as heatwave exacerbates pressure on services. BMJ 2018;362:k3492. doi: 10.1136/bmj.k3492 pmid: 30104239

13 Walker PGT, Whittaker C, Watson OJ, etal. The impact of COVID-19 and strategies for mitigation and suppression in low- and middle-income countries. Science 2020;369:413-22. doi: 10.1126/science.abc0035 pmid: 32532802

14 Rose G. Sick individuals and sick populations. Int J Epidemiol2001;30:427-32, discussion 433-4. doi: 10.1093/ije/30.3.427 pmid: 11416056

15 Jay O, Capon A, Berry P, etal. Reducing the health effects of hot weather and heat extremes: from personal cooling strategies to green cities. Lancet 2021;398:709-24. doi: 10.1016/S0140-6736(21)01209-5 pmid: 34419206

16 Mazzone A, Khosla R. Socially constructed or physiologically informed? Placing humans at the core of understanding cooling needs. Energy Res Soc Sci 2021;77:102088.doi: 10.1016/j.erss.2021.102088

17 Andrijevic M, Byers E, Mastrucci A, Smits J, Fuss S. Future cooling gap in shared socioeconomic pathways. Environ Res Lett 2021;16:94053.doi: 10.1088/1748-9326/ac2195

18 Davis L, Gertler P, Jarvis S, Wolfram C. Air conditioning and global inequality. Glob Environ Change 2021;69:102299.doi: 10.1016/j.gloenvcha.2021.102299

19 Hsu A, Sheriff G, Chakraborty T, Manya D. Disproportionate exposure to urban heat island intensity across major US cities. Nat Commun 2021;12:2721. doi: 10.1038/s41467-021-22799-5 pmid: 34035248

20 Nugent C. City heat is worse if you're not rich or white. Time2021. https://time.com/6078019/cityheat-climate-change-inequity/ 